\title{
Electron attachment to amino acid clusters in helium nanodroplets: Glycine, alanine, and serine
}

\author{
F. Ferreira da Silva, ${ }^{1}$ S. Denifl, ${ }^{1}$ T. D. Märk, ${ }^{1}$ A. M. Ellis, ${ }^{2, a)}$ and P. Scheier ${ }^{1, b)}$ \\ ${ }^{1}$ Institut für Ionenphysik und Angewandte Physik and Center of Molecular Biosciences Innsbruck, \\ Universität Innsbruck, Technikerstr. 25, A-6020 Innsbruck, Austria \\ ${ }^{2}$ Department of Chemistry, University of Leicester, University Road, Leicester LE1 7RH, United Kingdom
}

(Received 5 February 2010; accepted 23 April 2010; published online 4 June 2010)

\begin{abstract}
The first detailed study of electron attachment to amino acid clusters is reported. The amino acids chosen for investigation were glycine, alanine, and serine. Clusters of these amino acids were formed inside helium nanodroplets, which provide a convenient low temperature $(0.37 \mathrm{~K})$ environment for growing noncovalent clusters. When subjected to low energy $(2 \mathrm{eV})$ electron impact the chemistry for glycine and alanine clusters was found to be similar. In both cases, parent cluster anions were the major products, which contrasts with the corresponding monomers in the gas phase, where the dehydrogenated products $\left(\left[\mathrm{AA}_{n}-\mathrm{H}\right]^{-}\right.$, where $\mathrm{AA}=$ amino acid monomer) dominate. Serine clusters are different, with the major product being the parent anion minus an $\mathrm{OH}$ group, an outcome presumably conferred by the facile loss of an $\mathrm{OH}$ group from the $\beta$ carbon of serine. In addition to the bare parent anions and various fragment anions, helium atoms are also observed attached to both the parent anion clusters and the dehydrogenated parent anion clusters. Finally, we present the first anion yield spectra of amino acid clusters from doped helium nanodroplets as a function of incident electron energy. () 2010 American Institute of Physics. [doi:10.1063/1.3429743]
\end{abstract}

\section{INTRODUCTION}

Amino acid clusters have recently generated considerable interest. The most celebrated amino acid cluster derives from serine, which shows a remarkable tendency to produce octamers in high abundance. These octamers, in the form of cations (e.g., protonated octamers) or in association with anions, have been observed by mass spectrometry using electrospray ionization of solutions and related techniques, such as sonic spray ionization. ${ }^{1-4}$ These observations have been taken as strong evidence for a special stability of the neutral octamers. ${ }^{3}$ Furthermore, a comparison of chiral and racemic solutions of serine reveals a strong chiral preference at work in the cluster formation process. ${ }^{1}$ This in turn has led to speculation that the strong homochirality of serine clusters could have been the source of the well-known homochirality in key biological molecules in the emergence of life on Earth. $^{5}$

Another amino acid cluster that has recently generated interest is the dimer of glycine. Crystalline glycine has three known polymorphs, designated $\alpha, \beta$, and $\gamma$, but only the $\alpha$ polymorph tends to be formed by crystallization from $p H$ neutral aqueous solution. ${ }^{6,7}$ Explanations have been put forward for this structural preference which derive from the prior formation of cyclic dimers in solution, which seed the growth of the $\alpha$ polymorph in preference to the $\beta$ and $\gamma$ forms. Although this model has recently been challenged, ${ }^{8,9}$ an improved understanding of the interactions between

\footnotetext{
${ }^{a)}$ Electronic mail: andrew.ellis@le.ac.uk.

${ }^{\mathrm{b})}$ Electronic mail paul.scheier@uibk.ac.at
}

glycine molecules remains an important prerequisite for constructing a feasible mechanism for explaining polymorph selectivity.

Other evidence for the significance of amino acid clusters has emerged. For example, Charvat et al. have recorded mass spectra derived from laser-induced liquid beam ionization desorption (LILBID). This technique is an alternative to electrospray ionization for extracting ions from solutions and Charvat et al. ${ }^{10}$ have argued that LILBID comes close to reflecting the cluster distributions in the original solution. By investigating several amino acids and by varying solution conditions such as concentration and $p H$, amino acid clusters were readily observed and in some cases exceed the signal from monomer ions in the mass spectrum.

It has recently been established that low energy $(<20 \mathrm{eV})$ electrons can induce single and double strand breaks in DNA. ${ }^{11}$ This has motivated several recent studies of low energy electron attachment to the basic building blocks of proteins, the alpha amino acids. ${ }^{12-21}$ However, there have so far been no comparable studies of noncovalent clusters of amino acids. One of the interesting features of amino acids is the potential for competition between zwitterionic and nonzwitterionic tautomers. A good example of this is glycine, which is found only as the neutral tautomer in the gas phase ${ }^{22}$ whereas in aqueous solution and in the solid phase there is firm evidence that the zwitterion predominates. ${ }^{23,24}$ Zwitterionic forms become plausible in amino acid clusters because there is greater tolerance to charge separation when there are one or more neighboring amino acid monomers. In effect, the charge separation can be stabilized by solvation by the surrounding species. In turn, 

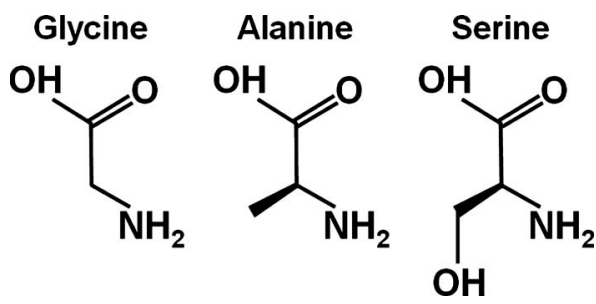

FIG. 1. Chemical structure of the amino acids glycine, alanine, and serine.

this could alter the response to electron attachment when the monomers are compared to the dimers. For this reason, and because of the potential biological significance of amino acid clusters described above, we report here the first detailed study of electron attachment to amino acid clusters. These clusters are formed in helium nanodroplets, which provide a convenient low temperature environment for growing noncovalent clusters. In this study glycine, alanine, and serine (the structures are shown in Fig. 1) have been targeted for investigation.

\section{EXPERIMENTAL}

The experimental measurements were carried out using a double focusing mass spectrometer coupled with a helium cluster source. The apparatus and basic operating procedure has already been described elsewhere, ${ }^{25,26}$ so only a brief account will be given here. Helium nanodroplets were formed by expansion of high purity helium into vacuum at high stagnation pressure through a pinhole nozzle $(5 \mu \mathrm{m}$ diameter). The nozzle was cooled to a low temperature and the mean size of the helium droplets is dictated by the combination of gas stagnation pressure and nozzle temperature. For the work described here, the helium stagnation pressure was 20 bar and the nozzle temperature was $11 \mathrm{~K}$, giving droplets with a mean size of roughly 13000 helium atoms. The droplet expansion was then skimmed and entered a pick-up region, where amino acid molecules were added by oven evaporation.

After picking up the amino acid, the doped droplets were then skimmed a second time and entered the source region of the mass spectrometer, where they were subjected to electron impact. The ion source is of the Nier-type with an energy resolution of $\sim 1 \mathrm{eV}$ and the current was set to $20 \mu \mathrm{A}$. The mass spectrometer is a modified two-sector field instrument (Varian-MAT CH5), which provides a mass resolution of $m / \Delta m=200$ with open slits.

Glycine was purchased from Sigma Aldrich ( $\geq 99 \%$ purity) while alanine and serine were both obtained from Fluka ( $\geq 99.5 \%$ purity). All were used without any further purification. In the case of alanine, a racemic mixture was used but for serine the $L$ enantiomer was employed because of its prior use in some earlier experiments.

\section{RESULTS AND DISCUSSION}

\section{A. Reaction products}

The clusters of all three amino acids considered in the present work show similar electron-induced chemistry. Glycine is considered first, and the negative ion mass spectrum

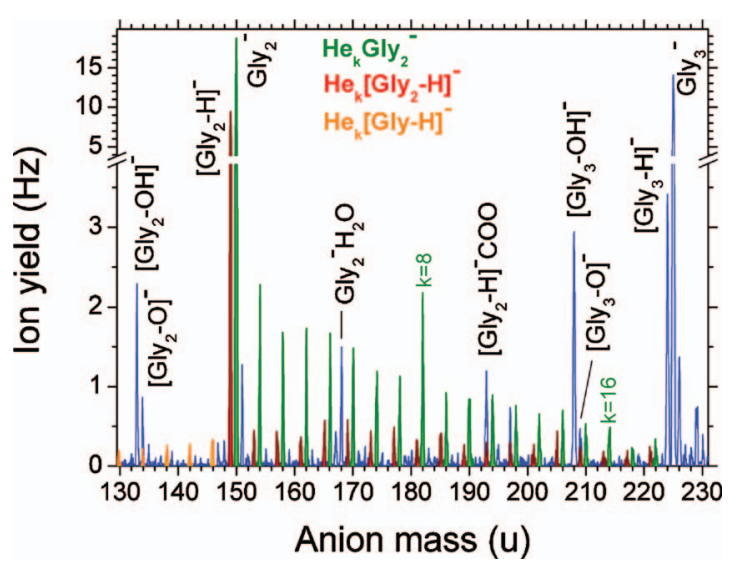

FIG. 2. Negative ion mass spectrum for glycine in helium nanodroplets. The electron impact energy was $\sim 2 \mathrm{eV}$. This mass spectrum focuses on the region between the dimer and trimer anions.

resulting from an incident electron impact energy of about 2 $\mathrm{eV}$ is shown in Fig. 2. This mass spectrum focuses mainly on the mass region between the dimer and trimer anions, which is also representative of the behavior for higher clusters. As in the case of acetic acid, ${ }^{27}$ the yield of the monomer anions of the presently investigated amino acids is more than an order of magnitude lower than the yield of the dimer and trimer cluster anions. Furthermore, the main species observed is the dehydrogenated parent anion, $[\mathrm{AA}-\mathrm{H}]^{-}$, and this is mainly due to gas phase amino acid molecules that leak into the ionization region of the mass spectrometer. Thus the monomer sections of the mass spectra are not shown in the present study.

In contrast to the monomer, the most intense peak for each particular cluster size is the parent cluster anion. Similar behavior has been reported previously from this laboratory in the case of thymine clusters, where parent cluster ions consisting of up to six monomer units were found to survive intact. ${ }^{25}$ Our current work reinforces the thymine observation and is clear evidence that embedding the amino acid clusters in liquid helium is able to prevent full conversion to the dehydrogenated species.

Nevertheless, dehydrogenated cluster anions are also produced and are the most abundant products after the parent cluster anions. Also significant, but rather weak, are peaks in the mass spectrum at $17 \mathrm{amu}$ below the parent anion peaks. This has been attributed to loss of $\mathrm{OH}$ in Fig. 2, but it could equally arise from loss of $\mathrm{NH}_{3}$. However, time consuming reactions, such as rearrangement processes, are often severely impeded in helium droplets. ${ }^{28,29}$ Consequently, $\mathrm{OH}$ ejection is the more likely channel giving rise to the peaks at $17 \mathrm{amu}$ below the parent anion. This peak has also been seen in previous gas phase studies of the electron attachment to glycine monomers. ${ }^{30-32}$

A few additional weak peaks can be identified in the mass spectrum of glycine which are attributed to the species $\mathrm{Gly}_{n}{ }^{-} \cdot \mathrm{H}_{2} \mathrm{O},\left[\mathrm{Gly}_{n}-\mathrm{H}\right]^{-} \cdot \mathrm{COO}$, and $\left[\mathrm{Gly}_{n}-\mathrm{O}\right]^{-}$. The first two species must arise from intracluster reactions induced by electron attachment. The assignment to $\left[\mathrm{Gly}_{n}-\mathrm{O}\right]^{-}$is tentative, since loss of $\mathrm{NH}_{2}$ instead of an $\mathrm{O}$ atom is possible and in the present study we have no evidence to distinguish be- 


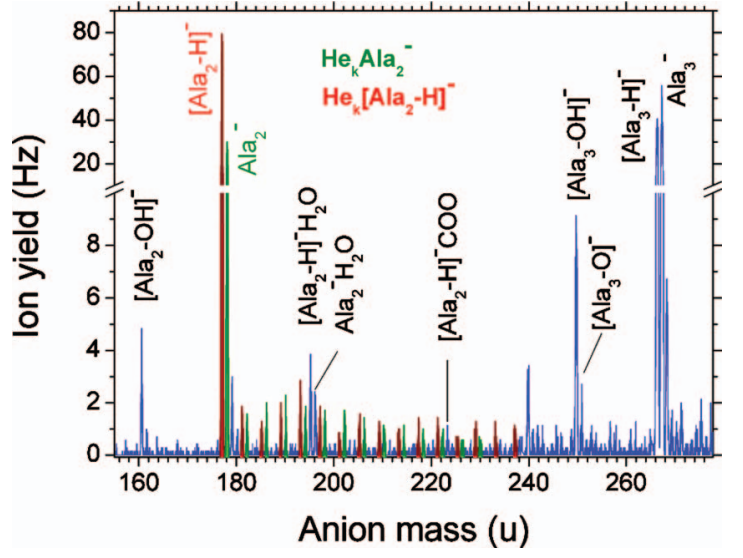

FIG. 3. Negative ion mass spectrum for alanine in helium nanodroplets showing the region near the dimer and trimer anion peaks. The electron impact was energy was $\sim 2 \mathrm{eV}$.

tween these two. Either way, no such product was found in electron attachment to the glycine monomer and this minor product is only produced in reactions of the cluster anions.

The anion chemistry for alanine clusters is broadly similar to that of glycine clusters, although there are differences in relative abundances of products. Figure 3 shows the relevant mass spectrum, which was recorded at the same incident electron energy as that of glycine clusters. In contrast to glycine, the parent anion is now less abundant than the dehydrogenated species. Other fragments observed for alanine clusters are essentially the same as for glycine clusters.

It is for serine clusters where the major difference is seen in reaction products. In this case, the $\mathrm{OH}$ loss product, $\left[\mathrm{S}_{n}-\mathrm{OH}\right]^{-}$, is now the major product anion for the trimer and larger clusters. Figures 4 and 5 illustrate this by showing the region between the dimer and trimer masses and a wider scan extending from the trimer to the pentamer, respectively. The situation changes for the dimer, where $\mathrm{OH}$ loss now becomes a minor channel (not shown in Fig. 4) and below the monomer there is no evidence of any fragmentation products other than $[\mathrm{S}-\mathrm{H}]^{-}$(which, as mentioned earlier, is more abundant than $\mathrm{S}^{-}$). Alanine differs from glycine in having a methyl group attached to the alpha carbon, whereas in serine this

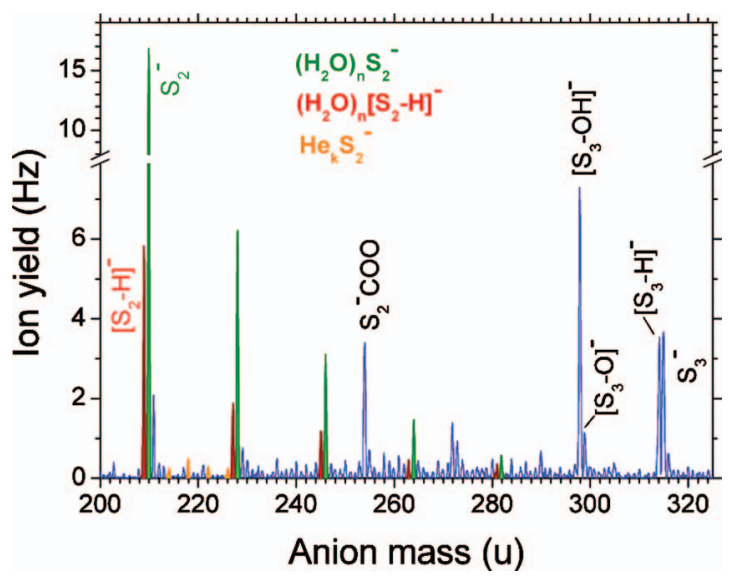

FIG. 4. Negative ion mass spectrum for serine in helium nanodroplets showing the rgion near the dimer and trimer anion peaks. The electron impact energy was $\sim 2 \mathrm{eV}$.

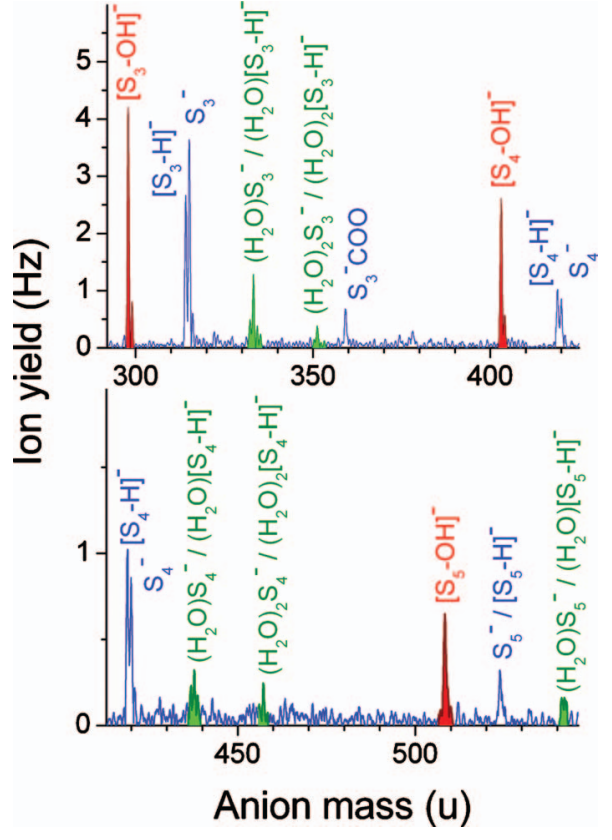

FIG. 5. Negative ion mass spectrum for serine in helium nanodroplets showing features near the trimer, tetramer and pentamer parent anion peaks.

methyl group is replaced by a hydroxymethyl $\left(\mathrm{CH}_{2} \mathrm{OH}\right)$ substituent. This difference presents serine with a facile leaving group that is not available to the other two amino acids. While this accounts for the greater prominence of $\mathrm{OH}$ loss for serine clusters, it does not explain why this channel only becomes predominant for the trimer and larger clusters. Although only speculation, we suggest that this might reflect the increasing likelihood of zwitterionic behavior in the serine clusters as they increase in size. Mass-selective infrared spectroscopic studies of protonated serine clusters suggest a progressive increase in the proportion of clusters with zwitterionic character as the size of the cluster increases. ${ }^{33}$ This is reflected in the progressive dominance of an absorption feature originating from a free $\mathrm{O}-\mathrm{H}$ group as the cluster size increases. In other words, in the non-zwitterionic form the $\mathrm{OH}$ group from the $\mathrm{CH}_{2} \mathrm{OH}$ unit is involved in hydrogen bonding, most probably to the $\mathrm{NH}_{2}$ group, but in the zwitterionic form this hydrogen bonding is disrupted. Consequently, according to this simple picture, unimolecular loss of $\mathrm{OH}$ is more likely from zwitterionic than non-zwitterionic isomers. If we assume that this also applies to the anions, then the change in chemical outcome for the dimer anion of serine, where $\mathrm{OH}$ loss is a very minor channel (not shown in Figs. 4 and 5), to the trimer, where $\mathrm{OH}$ loss is the major product channel, can be interpreted in terms of a switch from nonzwitterionic (dimer) to zwitterionic character (trimer). One would expect the zwitterionic character to be maintained for larger clusters and this is consistent with the observed dominance of the $\mathrm{OH}$ loss channel for the trimer through to the hexamer.

\section{B. Amino acid cluster anions with attached helium atoms}

In addition to the chemistry described above, anions with attached helium atoms are also observed. The formation 
of such clusters demonstrates the high degree of cooling of the anions, if not for all then certainly for a significant subset of the amino acid clusters produced in the current experiments.

The most prominent helium clusters are those formed with glycine dimer anions and the peaks are specifically identified in Fig. 2. $\mathrm{He}_{k} \mathrm{Gly}_{2}{ }^{-}$clusters are detectable with up to at least 18 helium atoms attached. Helium clusters are also seen with the dehydrogenated dimer anion, but the ratio of intensities compared with $\mathrm{He}_{k} \mathrm{Gly}_{2}{ }^{-}$is smaller than the intensity ratio of the bare $\left[\mathrm{Gly}_{2}-\mathrm{H}\right]^{-}$and $\mathrm{Gly}_{2}{ }^{-}$ions. This may reflect substantial differences in the electronic structures of the two anions, with $\mathrm{Gly}_{2}{ }^{-}$offering a more attractive binding environment for helium atoms.

In contrast to the cluster anions, the glycine monomer anion shows no and $[\mathrm{Gly}-\mathrm{H}]^{-}$shows very little evidence of attached helium atoms. Glycine is expected to have a negative electron affinity because there are no low-lying vacant orbitals on the molecule to accept the incoming electron. ${ }^{34}$ Consequently, the only way to attach and retain an electron is through a binding interaction with the molecular dipole moment. ${ }^{35} A b$ initio calculations have been carried out by Gutowski et al. ${ }^{36}$ on the possible structures of glycine monomer and its anion and the dipole moments of these species have been calculated. The most stable species is a neutral (i.e., nonzwitterionic) structure which is calculated to have a low dipole moment $(1.2 \mathrm{D})$. This is too small to allow formation of a dipole-bound anion. However, the calculations by Gutowski et al. $^{36}$ also show other isomers. There is a non-ionic but substantially more polar (dipole moment $\sim 5.5 \mathrm{D}$ ) isomer only $300 \mathrm{~cm}^{-1}$ or so above the global minimum, which seems a plausible precursor for the observed anion in the current experiments.

Although there are already several minima on the potential energy surface of glycine monomer, the potential energy landscape for the dimer is far more complex. In a joint experimental and theoretical study, Chocholoušová et al. ${ }^{37}$ identified the 22 lowest energy isomers. These include cyclic species, where the two carboxylic acid units form a ring structure with two hydrogen bonds, along with stacked structures and T-shaped structures (among others). Based on IR spectra of glycine dimer in helium nanodroplets, recorded in the same study, it seems likely that the predominant species in helium droplets is a metastable T-shaped isomer. Consequently, if that assignment proves correct then the same species would be expected to be formed under our experimental conditions. Whereas dipole binding is necessary to form an anion for the monomer, it is unclear if this is also the case for glycine clusters, since the solvation effect of adding one or more glycine molecules may create a bound valence state for the anion. If glycine clusters also form dipole-bound anions then there is no obvious reason why helium atoms should not also be observed attached to the monomer anions. However, if the clusters lead to valence anions then the preference for attaching helium atoms shown by the clusters may derive from the very different charge distribution of the cluster anions when compared to the monomer anion. Detailed theo-

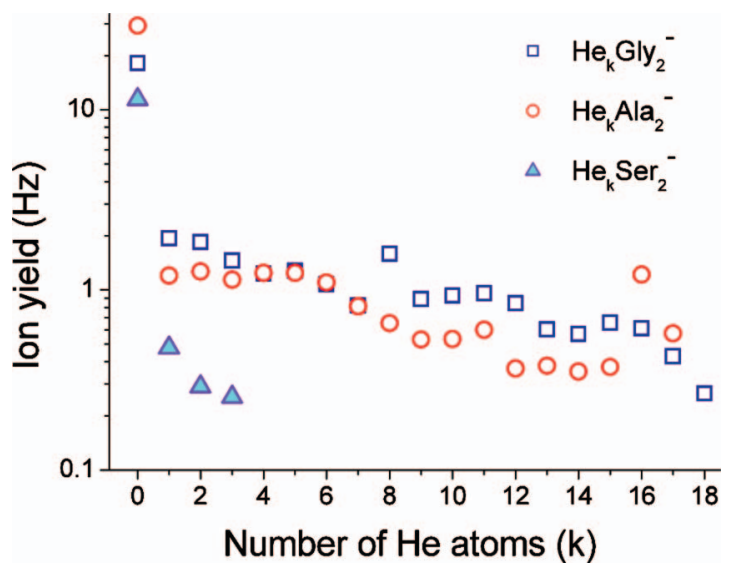

FIG. 6. Relative intensities (note the logarithmic scale) of the $\mathrm{He}_{k} \mathrm{AA}_{2}$ anions (AA=amino acid) for glycine, alanine, and serine.

retical work is necessary to understand the mechanism by which the helium atoms bind to amino acid anions and this is beyond the scope of the current work.

Interestingly, the $\mathrm{He}_{k} \mathrm{Gly}_{2}{ }^{-}$clusters appear to show a very prominent "magic" number peak at $k=8$ (see Fig. 1). There is also an indication of a shell closure at $k=16$. Magic number features in mass spectra are usually interpreted in terms of the enhanced stability of particular species, e.g., derived from the closure of specific structural shells. This may be responsible for the magic and antimagic features observed here but further theoretical work is essential for any detailed interpretation.

Like glycine, the monomer anions of alanine and serine show no evidence of any attached helium atoms in the mass spectra. However, helium atoms are seen attached to cluster anions of these two amino acids and peaks corresponding to these species are indicated in Figs. 3 and 5, but they are considerably less intense relative to the $\mathrm{He}$-free parent ions when compared to glycine. A summary of the relative abundances of helium clusters in the case of the parent dimer anion is presented in Fig. 6. This also includes the glycine dimer for comparison. The clusters of helium atoms with the serine dimer anion show no magic numbers, and indeed the anions with helium atoms become undetectably weak beyond $k=4$. In contrast the alanine dimer anion shows an extensive series of peaks arising from attached helium atoms with a pronounced magic number at $k=16$ but no magic number at $k=8$.

\section{Anion efficiency curves}

Figure 7 shows plots for the anion production efficiency as a function of electron kinetic energy, focusing on glycine dimer and glycine trimer parent anions. Similar plots were obtained for other glycine parent cluster anions, and indeed for parent cluster anions of alanine and serine, so here we focus solely on glycine since it is typical of the other amino acids. The anion efficiency curve is similar to that seen for the dehydrogenated monomer anion of amino acids in gas phase dissociative electron attachment studies. ${ }^{12-21}$ The low energy attachment reaches a peak maximum at approximately $1 \mathrm{eV}$ in the gas phase and leads exclusively to the 


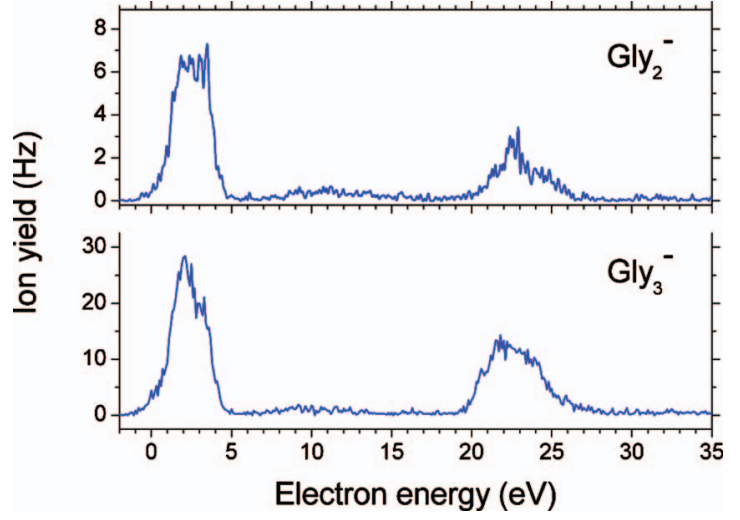

FIG. 7. Glycine dimer and trimer anion yield spectra as a function of incident electron kinetic energy.

dehydrogenated monomer anion, i.e., no parent monomer anion survives electron attachment. In other words, the initial anion formed is unstable on the timescale of the mass spectrometry experiment. However, as previously mentioned, in the current work a considerable proportion of the initially formed parent cluster anions do survive to detection, an observation attributed to rapid cooling by the helium, which partially quenches the dehydrogenation route The low energy resonance in the efficiency curves for the $\mathrm{AA}_{n}{ }^{-}$channel in helium nanodroplets is shifted approximately $1 \mathrm{eV}$ higher when compared to the gas phase because of the approximately $1 \mathrm{eV}$ threshold energy required for the electrons to penetrate the helium. ${ }^{25,28}$ The peak above $20 \mathrm{eV}$ is derived from inelastic scattering of the electrons by the helium. ${ }^{25}$

A higher energy resonance, peaking at $10 \mathrm{eV}$, becomes apparent for the dehydrogenated cluster anions of glycine, as can be seen in Fig. 8. This is in the region previously assigned to core-excited resonances in the glycine monomer, which lead to a variety of reaction channels. ${ }^{30-32}$ However, in helium nanodroplets the rapid cooling severely curtails these alternative reaction channels and many of the ions get funneled into the $\left[\mathrm{AA}_{n}-\mathrm{H}\right]^{-}$channel. Another noticeable feature is the variation in relative intensities of the first two resonances for each cluster in Fig. 8. This is partly a consequence of the reduction in size of the helium droplets by evaporative loss when incorporating larger clusters compared with

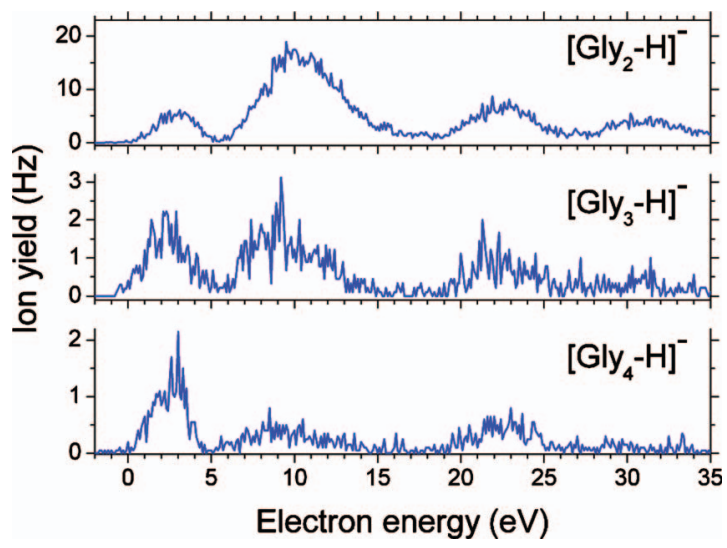

FIG. 8. The yield of dehydrogenated glycine cluster anions as a function of incident electron kinetic energy. smaller ones. Low energy electrons are readily scattered by helium and therefore many do not penetrate sufficiently far into the droplet to reach the amino acid cluster if the droplet is large. However, as the droplet shrinks in size the proportion of low energy electrons reaching the dopant increases and thus the intensity ratio responds accordingly. In addition, it is worth noting that bubble formation in helium droplets at very low electron energies reduces the electron mobility and thus enhances autodetachment. Consequently, this process distorts the ion yield profile by reducing the intensity of the lowest energy feature.

\section{CONCLUSIONS}

The effect of low energy electron impact on the clusters of three different amino acids (glycine, alanine, and serine) has been reported for the first time. The clusters were formed inside superfluid helium nanodroplets and anionic products ejected into the gas phase were detected by mass spectrometry. The chemistry of the clusters is different from the corresponding monomers. Whereas the dehydrogenated product dominates over the parent anion for the monomer, excitation by $1 \mathrm{eV}$ electrons for either glycine or alanine clusters gave the parent cluster anions as the major reaction products. However, there were also significant fragmentation channels, such as $\mathrm{OH}$ loss from the parent anion. In serine this fragmentation becomes more severe and the major product is the $\left[\mathrm{AA}_{n}-\mathrm{OH}\right]^{-}$channel. The differences between glycine and alanine on the one hand, and serine on the other, are assumed to arise from the presence of a facile leaving group $(\mathrm{OH})$ located on the $\beta$ carbon atom in the case of serine.

Peaks are also observed in the mass spectra which arise from the amino acid cluster anions with multiple helium atoms attached. Surprisingly, helium atoms are only seen attached to amino acid clusters, i.e., the corresponding monomer anions show no propensity for helium attachment. For both glycine and alanine, the relative abundance profiles show magic numbers of helium atoms, although these magic numbers are not comparable in the two cases. Once again serine clusters are different and show only a modest willingness to attach helium atoms and give no magic numbers.

The anion yield spectra of the amino acid clusters are similar to the corresponding spectra from dehydrogenated monomer anion channel in gas phase work. This is explained by the rapid cooling of the cluster anions by the liquid helium after electron attachment, which helps to prevent extensive dissociation into the $\left[\mathrm{AA}_{n}-\mathrm{H}\right]^{-}$channel.

\section{ACKNOWLEDGMENTS}

This work was supported by the FWF (Grant No. P19073) and the European Commission, Brussels. S.D. gratefully acknowledges an APART grant from the Austrian Academy of Sciences. A.M.E. is grateful for the support of a visit to Innsbruck by ECCL COST Action CM0601.

\footnotetext{
${ }^{1}$ R. G. Cooks, D. Zhang, K. J. Koch, F. C. Gozzo, and M. N. Eberlin, Anal. Chem. 73, 3646 (2001).

${ }^{2}$ K. Yamaguchi, J. Mass Spectrom. 38, 473 (2003).

${ }^{3}$ K. J. Koch, F. C. Gozzo, D. Zhang, M. N. Eberlin, and R. G. Cooks, Chem. Commun. (Cambridge) 2001, 1854.
} 
${ }^{4}$ Z. Takats, S. C. Nanita, R. G. Cooks, G. Schlosser, and K. Vekey, Anal Chem. 75, 1514 (2003).

${ }^{5}$ S. C. Nanita and R. G. Cooks, Angew. Chem., Int. Ed. 45, 554 (2006).

${ }^{6}$ G. L. Perlovich, L. K. Hansen, and A. Bauer-Brandl, J. Therm Anal Calorim. 66, 699 (2001).

${ }^{7}$ C. S. Towler, R. J. Davey, R. W. Lancaster, and C. J. Price, J. Am. Chem. Soc. 126, 13347 (2004)

${ }^{8}$ S. Hamad, C. E. Hughes, C. R. A. Catlow, and K. D. M. Harris, J. Phys. Chem. B 112, 7280 (2008).

${ }^{9}$ J. Huang, T. C. Stringfellow, and L. Yu, J. Am. Chem. Soc. 130, 13973 (2008).

${ }^{10}$ A. Charvat, E. Lugovoj, M. Faubel, and B. Abel, Eur. Phys. J. D 20, 573 (2002).

${ }^{11}$ B. Boudaïffa, P. Cloutier, D. Hunting, M. A. Huels, and L. Sanche, Science 287, 1658 (2000).

${ }^{12}$ I. Bald, E. Kopyra, and E. Illenberger, Angew. Chem., Int. Ed. 45, 4851 (2006)

${ }^{13}$ S. Denifl, S. Ptasinska, M. Cingel, S. Matejcik, P. Scheier, and T. D. Märk, Chem. Phys. Lett. 377, 74 (2003).

${ }^{14}$ S. Ptasinska, S. Denifl, P. Scheier, E. Illenberger, and T. D. Märk, Angew. Chem., Int. Ed. 44, 6941 (2005).

${ }^{15}$ S. Denifl, P. Sulzer, D. Huber, F. Zappa, M. Probst, T. D. Märk, P. Scheier, N. Injan, J. Limtrakul, R. Abouaf, and H. Dunet, Angew. Chem. Int. Ed. 46, 5238 (2007).

${ }^{16}$ H. D. Flosadóttir, S. Denifl, F. Zappa, N. Wendt, A. Mauracher, A. Bacher, H. Jonsson, T. D. Märk, P. Scheier, and O. Ingolfsson, Angew. Chem., Int. Ed. 46, 8057 (2007).

${ }^{17}$ S. Ptasinska, S. Denifl, V. Grill, T. Märk, P. Scheier, S. Gohlke, M. Huels, and E. Illenberger, Angew. Chem., Int. Ed. 44, 1657 (2005).

${ }^{18}$ S. Ptasińska, S. Denifl, V. Grill, T. D. Märk, E. Illenberger, and P. Scheier, Phys. Rev. Lett. 95, 093201 (2005).

${ }^{19}$ S. Denifl, P. Sulzer, F. Zappa, S. Moser, B. Krautler, O. Echt, D. K. Bohme, T. D. Märk, and P. Scheier, Int. J. Mass. Spectrom. 277, 296 (2008)

${ }^{20}$ P. Sulzer, E. Alizadeh, A. Mauracher, T. D. Märk, and P. Scheier, Int. J Mass. Spectrom. 277, 274 (2008).
${ }^{21}$ D. Huber, M. Beikircher, S. Denifl, F. Zappa, S. Matejcik, A. Bacher, V. Grill, T. D. Märk, and P. Scheier, J. Chem. Phys. 125, 084304 (2006).

${ }^{22}$ C. V. Kumar and A. Chaudhari, J. Am. Chem. Soc. 122, 830 (2000).

${ }^{23}$ P. G. Jönsson and A. Kvick, Acta Crystallogr., Sect. B: Struct. Crystallogr. Cryst. Chem. 28, 1827 (1972).

${ }^{24}$ F. R. Tortonda, J. L. Pascual-Ahuir, E. Silla, and I. Tunon, Chem. Phys. Lett. 260, 21 (1996).

${ }^{25}$ S. Denifl, F. Zappa, I. Mähr, J. Lecointre, M. Probst, T. D. Märk, and P. Scheier, Phys. Rev. Lett. 97, 043201 (2006).

${ }^{26}$ S. Denifl, F. Zappa, I. Mähr, A. Mauacher, M. Probst, T. D. Märk, and P. Scheier, J. Am. Chem. Soc. 130, 5065 (2008).

${ }^{27}$ F. Ferreira da Silva, S. Jaksch, G. Martins, H. M. Dang, M. Dampc, S. Denifl, T. D. Märk, P. Limao-Vieira, J. Liu, S. Yang, A. M. Ellis, and P. Scheier, Phys. Chem. Chem. Phys. 11, 11631 (2009).

${ }^{28}$ S. Denifl, F. Zappa, A. Mauracher, F. Ferreira da Silva, A. Bacher, O. Echt, T. D. Märk, D. K. Bohme, and P. Scheier, ChemPhysChem 9, 1387 (2008).

${ }^{29}$ S. Yang, S. M. Brereton, M. D. Wheeler, and A. M. Ellis, J. Phys. Chem. A 110, 1791 (2006).

${ }^{30}$ S. Gohlke, A. Rosa, E. Illenberger, F. Bruning, and M. A. Huels, J. Chem. Phys. 116, 10164 (2002).

${ }^{31}$ S. Ptasinska, S. Denifl, A. Abedi, P. Scheier, and T. D. Märk, Anal. Bioanal. Chem. 377, 1115 (2003).

${ }^{32}$ A. Mauracher, S. Denifl, A. Aleem, N. Wendt, F. Zappa, P. Cicman, M. Probst, T. D. Märk, P. Scheier, H. D. Flosadottir, O. Ingolfsson, and E. Illenberger, Phys. Chem. Chem. Phys. 9, 5680 (2007).

${ }^{33}$ X. Kong, I.-A. Tsai, S. Sabu, C.-C. Han, Y. T. Lee, H.-C. Chang, S.-Y. Tu, A. H. Kung, and C.-C. Wu, Angew. Chem., Int. Ed. 45, 4130 (2006).

${ }^{34}$ E. G. Diken, N. I. Hammer, and M. A. Johnson, J. Chem. Phys. 120, 9899 (2004)

${ }^{35}$ M. Gutowski, P. Skurski, and J. Simons, J. Am. Chem. Soc. 122, 10159 (2000).

${ }^{36}$ J. Simons, J. Phys. Chem. 112, 6401 (2008).

${ }^{37}$ J. Chocholoušová, J. Vacek, F. Huisken, O. Werhahn, and P. Hobza, J. Phys. Chem. A 106, 11540 (2002). 